\title{
Coupled-Mode Theory for Guided-Wave Optics
}

\author{
AMNON YARIV
}

\begin{abstract}
The problem of propagation and interaction of optical radiation in dielectric waveguides is cast in the coupled-mode formalism. This approach is useful for treating problems involving energy exchange between modes. A derivation of the general theory is followed by application to the specific cases of electrooptic modulation, photoelastic and magnetooptic modulation, and optical filtering. Also treated are nonlinear optical applications such as second-harmonic generation in thin films and phase matching.
\end{abstract}

\section{INTRODUCTION}

A GROWING BODY of theoretical and experimental work has been recently building up in the area of guided-wave optics, which may be defined as the study and utilization of optical phenomena in thin dielectric waveguides [1], [2]. Some of this activity is due to the hopes for integrated optical circuits in which a number of optical functions will be performed on small solid substrates with the interconnections provided by thin-film dielectric waveguides [3], [4]. Another reason for this interest is the possibility of new nonlinear optical devices and efficient optical modulators which are promised by this approach [5]-[7].

A variety of theoretical ad hoc formalisms have been utilized to date in treating the various phenomena of guidedwave optics. In this paper we present a unified theory cast in the coupled-mode form to describe a large number of seemingly diverse phenomena. These include: 1) nonlinear optical interactions; 2 ) phase matching by periodic perturbations; 3) electrooptic switching and modulation; 4) photoelastic switching and modulation; and 5) optical filtering and reflection by a periodic perturbation.

\section{The Coupled-Mode Formalism}

We will employ, in what follows, the coupled-mode formalism [8] to treat the various phenomena listed in Section I. Before embarking on a detailed analysis it will prove beneficial to consider some of the common features of this theory. Consider two electromagnetic modes with, in general, different frequencies whose complex amplitudes are $A$ and $B$. These are taken as the eigenmodes of the unperturbed medium so that they represent propagating disturbances

$$
a(z, x, t)=A e^{i\left(\omega_{a} t=\beta_{u} z\right\rangle} f_{a}(x)
$$

Manuscript received March 9, 1973. This research was supported in part by the National Science Foundation and in part by the Advanced Research Projects Agency through the Army Research Office, Durham, N.C.

The author is with the Department of Electrical Engineering, California Institute of Technology, Pasadena, Calif. 91109.

$$
b(z, x, t)=B e^{i\left(\omega h l+\beta_{h z}\right)} f_{b}(x)
$$

with $A$ and $B$ constant.

In the presence of a perturbation which, as an example, can take the place of a periodic electric field, a sound wave, or a surface corrugation, power is exchanged between modes $a$ and $b$. The complex amplitudes $A$ and $B$ in this case are no longer constant but will be found to depend on $z$. They will be shown below to obey relations of the type

$$
\begin{aligned}
& \frac{d A}{d z}=\kappa_{a b} B e^{-i \Delta z} \\
& \frac{d B}{d z}=\kappa_{b a} A e^{+i \Delta z}
\end{aligned}
$$

where the phase-mismatch constant $\Delta$ depends on the propagation constants $\beta_{a}$ and $\beta_{b}$ as well as on the spatial variation of the coupling perturbation. The coupling coefficients $\kappa_{a b}$ and $\kappa_{b a}$ are determined by the physical situation under consideration and their derivation will take up a major part of this paper. Before proceeding, however, with the specific experimental situations, let us consider some general features of the solutions of the coupled-mode equations.

\section{A. Codirectional Coupling}

We take up, first, the case where modes $a$ and $b$ carry (Poynting) power in the same direction. It is extremely convenient to define $A$ and $B$ in such a way that $|A(z)|^{2}$ and $|B(z)|^{2}$ correspond to the power carried by modes $a$ and $b$, respectively. The conservation of total power is thus expressed as

$$
\frac{d}{d z}\left(|A|^{2}+|B|^{2}\right)=0
$$

which, using (2), is satisfied when [9]

$$
\kappa_{a b}=-\kappa_{b a}{ }^{*} .
$$

If boundary conditions are such that a single mode, say $b$, is incident at $z=0$ on the perturbed region $z>0$, we have

$$
b(0) \equiv B_{0}, \quad a(0)=0 .
$$

Subject to these conditions the solutions of (2) become

$$
A(z)=B_{0} \frac{2 \kappa_{a b}}{\left(4 \kappa^{2}+\Delta^{2}\right)^{1 / 2}} e^{-i \Delta z / 2} \sin \left[\frac{1}{2}\left(4 \kappa^{2}+\Delta^{2}\right)^{1 / 2} z\right]
$$




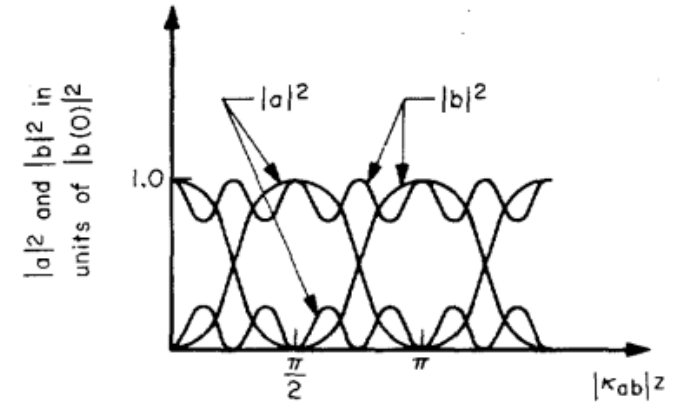

Fig. 1. The variation of the mode power in the case of codirectional coupling for phase-matched and unmatched operation.

$$
\begin{aligned}
B(z)=B_{0} e^{i \Delta z / 2}\left\{\cos \left[\frac{1}{2}\left(4 \kappa^{2}+\Delta^{2}\right)^{1 / 2} z\right]\right. \\
\left.\quad-i \frac{\Delta}{\left(4 \kappa^{2}+\Delta^{2}\right)^{1 / \overline{2}}} \sin \left[\frac{1}{2}\left(4 \kappa^{2}+\Delta^{2}\right)^{1 / 2} z\right]\right\}
\end{aligned}
$$

where $\kappa^{2} \equiv\left|\kappa_{a b}\right|^{2}$. Under phase-matched condition $\Delta=0$, a complete spatially periodic power transfer between modes $a$ and $b$ takes place with a period $\pi / 2 \kappa$.

$$
\begin{aligned}
& a(z, t)=B_{0} \frac{\kappa_{a b}}{\kappa} e^{i\left(\omega_{a} t-\beta_{a}\right)} \sin (\kappa z) \\
& b(z, t)=B_{0} e^{i\left(\omega_{b} t-\beta_{b z}\right)} \cos (\kappa z) .
\end{aligned}
$$

A plot of the mode intensities $|a|^{2}$ and $|b|^{2}$ is shown in Fig. 1. This figure demonstrates the fact that for phase mismatch $\Delta \gg\left|\kappa_{a b}\right|$ the power exchange between the modes is negligible. Specific physical situations which are describable in terms of this picture will be discussed further below.

\section{B. Contradirectional Coupling}

In this case the propagation in the unperturbed medium is described by

$$
\begin{aligned}
& a=A e^{i\left(\omega_{a} t+\beta_{a} z\right)} \\
& b=B e^{i\left(\omega_{b} t-\beta_{b z}\right)}
\end{aligned}
$$

where $A$ and $B$ are constant. Mode $a$ corresponds to a left $(-z)$ traveling wave while $b$ travels to the right. A time-space periodic perturbation can lead to power exchange between the modes. Conservation of total power can be expressed as

$$
\frac{d}{d z}\left(|A|^{2}-|B|^{2}\right)=0
$$

which is satisfied by (2) if we take

$$
\kappa_{a b}=\kappa_{b a}{ }^{*}
$$

so that

$$
\frac{d A}{d z}=\kappa_{a b} B e^{-i \Delta z} \quad \frac{d B}{d z}=\kappa_{a b} * A e^{i \Delta z} .
$$

In this case we take the mode $b$ with an amplitude $B(0)$ to be incident at $z=0$ on the perturbation region which occupies

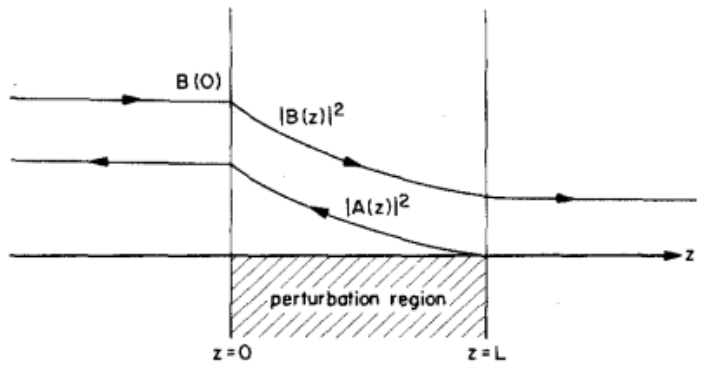

Fig. 2. The transfer of power from an incident forward wave $B(z)$ to a reflected wave $A(z)$ in the case of contradirectional coupling.

the space between $z=0$ and $z=L$. Since mode $a$ is generated by the perturbation we have $a(L)=0$. With these boundary conditions the solution of (11) is given by

$$
\begin{gathered}
A(z)=B(0) \frac{2 i \kappa_{a b} e^{-i(\Delta z / 2)}}{-\Delta \sinh \frac{S L}{2}+i S \cosh \frac{S L}{2}} \sinh \left[\frac{S}{2}(z-L)\right] \\
B(z)=B(0) \frac{e^{i(\Delta z / 2)}}{-\Delta \sinh \frac{S L}{2}+i S \cosh \frac{S L}{2}} \\
\cdot\left\{\Delta \sinh \left[\frac{S}{2}(z-L)\right]+i S \cosh \left[\frac{S}{2}(z-L)\right]\right\} \quad \text { (12) } \\
S \equiv \sqrt{4 \kappa^{2}-\Delta^{2}}, \quad \kappa \equiv\left|\kappa_{a b}\right| .
\end{gathered}
$$

Under phase-matching conditions $\Delta=0$ we have

$$
\begin{aligned}
& A(z)=B(0)\left(\frac{\kappa_{a b}}{\kappa}\right) \frac{\sinh [\kappa(z-L)]}{\cosh (\kappa L)} \\
& B(z)=B(0) \frac{\cosh [\kappa(z-L)]}{\cosh (\kappa L)} .
\end{aligned}
$$

A plot of the mode powers $|B(z)|^{2}$ and $|A(z)|^{2}$ for this case is shown in Fig. 2. For sufficiently large arguments of the cosh and sinh functions in (14), the incident-mode power decays exponentially along the perturbation region. This decay, however, is due not to absorption but to reflection of power into the backward traveling mode $a$. This case will be considered in detail in following sections, where acoustooptic, electrooptic, and spatial index perturbation will be treated. The exponential-decay behavior of Fig. 2 will be shown in Section VIII to correspond to the stopband region of periodic optical media.

\section{Electromagnetic Derivations of the Coupled- Mode Equations}

\section{A. TE Modes}

Consider the dielectric waveguide sketched in Fig. 3. It consists of a film of thickness $t$ and index of refraction $n_{2}$ sandwiched between media with indices $n_{1}$ and $n_{3}$. Taking $(\partial / \partial y)=0$, this guide can, in the general case, support a 


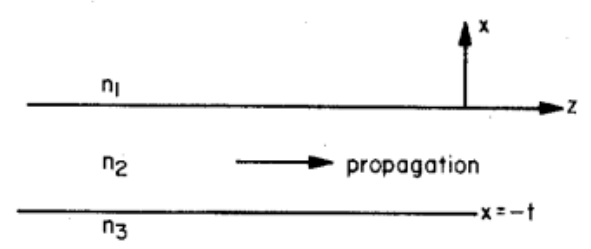

Fig. 3. The basic configuration of a slab dielectric waveguide.

finite number of confined TE modes with field components $E_{y}, H_{x}$, and $H_{z}$, and TM modes with components $H_{y}, E_{x}$, and $E_{z}$. The "radiation" modes of this structure which are not confined to the inner layer are not considered in this paper and will be ignored. The field component $E_{y}$ of the TE modes, as an example, obeys the wave equation

$$
\nabla^{2} E_{\nu}=\frac{n_{i}^{2}}{c^{2}} \frac{\partial^{2} E_{y}}{\partial t^{2}}, \quad i=1,2,3
$$

We take $E_{y}(x, z, t)$ in the form

$$
E_{y}(x, z, t)=\varepsilon_{y}(x) e^{l(\omega t-\beta z)} .
$$

The transverse function $\varepsilon_{y}(x)$ is taken as

$$
\mathcal{E}_{y}(x)=\left\{\begin{array}{lr}
C \exp (-q x), & 0 \leq x<\infty \\
C[\cos (h x)-(q / h) \sin (h x)], & -t \leq x \leq 0 \\
C[\cos (h t)+(q / h) \sin (h t)] & \exp [p(x+t)], \\
-\infty<x \leq-t
\end{array}\right.
$$

which, applying (15) to regions $1,2,3$, yields

$$
\begin{aligned}
h & =\left(n_{2}{ }^{2} k^{2}-\beta^{2}\right)^{1 / 2} \\
q & =\left(\beta^{2}-n_{1}{ }^{2} k^{2}\right)^{1 / 2} \\
p & =\left(\beta^{2}-n_{3}{ }^{2} k^{2}\right)^{1 / 2} \\
k & \equiv \omega / c .
\end{aligned}
$$

From the requirement that $E_{y}$ and $H_{z}$ be continuous at $x=0$ and $x=-t$, we obtain ${ }^{1}$

$$
\tan (h t)=\frac{q+p}{h\left(1-\frac{p q}{h^{2}}\right)} .
$$

This equation in conjunction with (18) is used to obtain the eigenvalues $\beta$ of the confined TE modes.

The constant $C$ appearing in (17) is arbitrary. We choose it in such a way that the field $\varepsilon_{y}(x)$ in (17) corresponds to a power flow of $1 \mathrm{~W}$ (per unit width in the $y$ direction) in the mode. A mode whose $E_{y}=A \varepsilon_{y}(x)$ will thus correspond to a

${ }^{1}$ The assumed form of $E_{y}$ in (17) is such that $\varepsilon_{y}$ and $\mathcal{H C}_{z}=(i / \omega \mu)$ $\partial \varepsilon_{y} / \partial x$ are continuous at $x=0$ and that $\varepsilon_{\nu}$ is continuous at $x=-t$. All that is left is to require continuity of $\partial \varepsilon_{y} / \partial x$ at $x=-t$. This leads to (19). power flow of $|A|^{2} \mathrm{~W} / \mathrm{m}$. The normalization condition is thus

$$
-\frac{1}{2} \int_{-\infty}^{\infty} E_{y} H_{x}{ }^{*} d x=\frac{\beta_{m}}{2 \omega \mu} \int_{-\infty}^{\infty}\left[\mathcal{E}_{y}{ }^{(m)}(x)\right]^{2} d x=1
$$

where the symbol $m$ denotes the $m$ th confined TE mode corresponding to $m$ th eigenvalue of (19).

Using (17) in (20) we determine

$$
C_{m}=2 h_{m}\left[\frac{\omega \mu}{\left|\beta_{m}\right|\left(t+\frac{1}{q_{m}}+\frac{1}{p_{m}}\right)\left(h_{m}{ }^{2}+{q_{m}}^{2}\right)}\right]^{1 / 2} .
$$

Since the modes $\varepsilon_{y}{ }^{(m)}$ are orthogonal we have

$$
\int_{-\infty}^{\infty} \varepsilon_{\nu}{ }^{(l)} \mathcal{E}_{y}{ }^{(m)} d x=\frac{2 \omega \mu}{\beta_{m}} \delta_{l, m} .
$$

\section{B. TM Modes}

The field components are

$$
\begin{aligned}
& H_{y}(x, z, t)=\mathfrak{K}_{y}(x) e^{i(\omega t-i \beta z)} \\
& E_{x}(x, z, t)=\frac{i}{\omega \epsilon} \frac{\partial H_{y}}{\partial z}=\frac{\beta}{\omega \epsilon} \mathcal{K}_{y}(x) e^{i(\omega t-\beta z)} \\
& E_{z}(x, z, t)=-\frac{i}{\omega \epsilon} \frac{\partial H_{y}}{\partial x}
\end{aligned}
$$

The transverse function $\mathfrak{H C}_{y}(x)$ is taken as

$$
{ } \mathcal{C}_{\nu}(x)=\left\{\begin{array}{lc}
-C\left[\frac{h}{\bar{q}} \cos (h t)+\sin (h t)\right] e^{p(x+t)}, & x<-t \\
C\left[-\frac{h}{\bar{q}} \cos (h x)+\sin (h x)\right], & -t<x<0 \\
-\frac{h}{\bar{q}} C e^{-q x}, & x>0 .
\end{array}\right.
$$

The continuity of $H_{y}$ and $E_{z}$ at the interfaces requires that the various propagation constants obey the eigenvalue equation

$$
\tan (h t)=\frac{h(\bar{p}+\bar{q})}{h^{2}-\bar{p} \bar{q}}
$$

where

$$
\bar{p} \equiv \frac{n_{2}{ }^{2}}{n_{3}{ }^{2}} p, \quad \bar{q} \equiv \frac{n_{2}{ }^{2}}{n_{1}{ }^{2}} q .
$$

The normalization constant $C$ is chosen so that the field represented by (23) and (24) carries $1 \mathrm{~W}$ per unit width in the $y$ direction.

$$
\frac{1}{2} \int_{-\infty}^{\infty} H_{\nu} E_{x}^{*} d x=\frac{\beta}{2 \omega} \int_{-\infty}^{\infty} \frac{\mathfrak{F C}_{y}{ }^{2}(x)}{\epsilon} d x=1
$$


or using $n_{i}{ }^{2} \equiv \epsilon_{i} / \epsilon_{0}$

$$
\int_{-\infty}^{\infty} \frac{\left[\mathcal{F}_{y}^{(m)}(x)\right]^{2}}{n^{2}(x)} d x=\frac{2 \omega \epsilon_{0}}{\beta_{m}} .
$$

This condition determines the value of $C_{m}$ as [10]

$$
\begin{aligned}
C_{m} & =2 \sqrt{\frac{\omega \epsilon_{0}}{\beta_{m} t_{\mathrm{eff}}}} \\
t_{\mathrm{eff}} & \equiv \frac{\bar{q}^{2}+h^{2}}{\bar{q}^{2}}\left[\frac{t}{n_{2}{ }^{2}}+\frac{q^{2}+h^{2}}{\bar{q}^{2}+h^{2}} \frac{1}{n_{1}{ }^{2} q}+\frac{p^{2}+h^{2}}{\bar{p}^{2}+h^{2}} \frac{1}{n_{3}{ }^{2} p}\right] .
\end{aligned}
$$

\section{The Coupling Equation}

The wave equation obeyed by the unperturbed modes is

$$
\nabla^{2} \mathbf{E}(\mathbf{r}, t)=\mu \epsilon \frac{\partial^{2} \mathbf{E}}{\partial t^{2}} .
$$

We will show below that in most of the experiments of interest to us we can represent the perturbation as a distributed polarization source $\mathbf{P}_{\text {pert }}(\mathbf{r}, t)$, which accounts for the deviation of the medium polarization from that which accompanies the unperturbed mode. The wave equation for the perturbed case follows directly from Maxwell's equations if we take D $=\epsilon_{0} \mathbf{E}+\mathbf{P}$.

$$
\nabla^{2} E_{\nu}(\mathrm{x}, t)=\mu \epsilon \frac{\partial^{2} E_{v}}{\partial t^{2}}+\mu \frac{\partial^{2}}{\partial t^{2}}\left[P_{\text {pert }}(\mathrm{r}, t)\right]_{\nu}
$$

with similar equations for the remaining Cartesian components of $\mathbf{E}$.

We may take the eigenmodes of (28) as an orthonormal set in which to expand $E_{y}$ and write

$$
\begin{aligned}
E_{y}=\sum_{i} \frac{A_{l}(z)}{2} \varepsilon_{y}{ }^{(l)}(x) e^{i\left(\omega t-\beta_{i z}\right)}+\mathrm{c} \cdot \mathrm{c} . \\
+\int_{\substack{n_{z}<n_{2} k \\
n_{3} k}} \frac{A(\beta)}{2} e^{i\left(\omega t-\beta_{z}\right)} \mathcal{E}_{\nu}{ }^{\langle\beta\rangle}(x) d \beta
\end{aligned}
$$

where $l$ extends over the discrete set of confined modes and includes both positive and negative traveling waves. The integration over $\beta$ takes in the continuum of radiation modes, and c.c. denotes complex conjugation. Our chief interest lies in perturbations which couple only discrete modes so that, in what follows, we will neglect the second term on the right side of (30). Problems of coupling to the radiation modes arise in connection with waveguide losses [11] and grating couplers [12].

Substituting (30) into (29), assuming "slow" variation so that $d^{2} A_{m} / d z^{2}<<\beta_{m} d A_{m} / d z$, and recalling that $\varepsilon_{y}{ }^{(m)}(x)$ $e^{i\left(\omega t-\beta_{m z}\right)}$ obeys the unperturbed wave equation (28), gives

$$
\sum_{l}\left[-i \beta_{l} \frac{d A_{l}}{d z} \delta_{\nu}{ }^{(l)}(x) e^{i\left(\omega t-\beta_{l}\right)}\right]+\text { c.c. }=\mu \frac{\partial^{2}}{\partial t^{2}}\left(P_{\mathrm{De} \tau t}\right)_{\nu} .
$$

Multiplying (31) by $\varepsilon_{y}^{(m)}(x)$, and integrating and making use of the orthogonality relation (22) yields

$$
\begin{array}{r}
\frac{d A_{m}{ }^{(-)}}{d z} e^{i\left(\omega t+\beta_{m} z\right)}-\frac{d A_{m}^{(+)}}{d z} e^{i\left(\omega t-\beta_{m z}\right)}+\text { c.c. } \\
=\frac{-i}{2 \omega} \frac{\partial^{2}}{\partial t^{2}} \int_{-\infty}^{\infty}\left[P_{\text {Dert }}(\mathbf{r}, t)\right]_{\nu} \varepsilon_{v}{ }^{(m)}(x) d x
\end{array}
$$

where $A_{m}^{(-)}$is the complex normal mode amplitude of the negative traveling TE mode while $A_{m}^{(+)}$is that of the positive one. Equation (32) is the main starting point for the following discussion in which we will consider a number of special cases.

\section{NONLINEAR INTERACTIONS}

In this section we consider the exchange of power between three modes of different frequencies brought about through the nonlinear optical properties of the guiding or bounding layers. The relevant experimental situations involve secondnarmonic generation, frequency up-conversion, and optical parametric oscillation. To be specific we consider first the case of second-harmonic generation from an input mode at $\omega / 2$ to an output mode at $\omega$. The perturbation polarization is taken as

$$
P_{i}{ }^{(\omega)}(\mathbf{r}, t)=\frac{1}{2}\left[P_{i}{ }^{(\omega)} e^{i(\omega t-\beta z)}+\text { c.c. }\right] .
$$

The complex amplitude of the polarization is

$$
P_{i}^{(\omega)}=d_{i j k}^{(\omega)}(\mathrm{r}) E_{j}^{\omega / 2} E_{k}^{\omega / 2}
$$

where $d_{i j k}{ }^{(\omega)}$ is an element of the nonlinear optical tensor and summation over repeated indices is understood. We have allowed, in (34), for a possible dependence of $d_{i j k}$ on the position $\boldsymbol{r}$.

\section{A. Case I: $T E_{\text {input }}-T E_{\text {output }}$}

Without going, at this point, into considerations involving crystalline orientation, let us assume that an optical field parallel to the waveguide $y$ direction will generate a second-harmonic polarization along the same direction

$$
P_{y}^{(\omega)}=d E_{y}^{(\omega / 2)} E_{y}{ }^{(\omega / 2)}
$$

where $P$ and $E$ represent complex amplitudes, and $d$ corresponds to a linear combination of $d_{i j k}$ which depends on the crystal orientation. In this special case an input TE mode at $\omega / 2$ will generate an output TE mode at $\omega$. Using (30) in (35) gives

$$
\begin{aligned}
& P_{\nu}(\mathrm{r}, t)=\frac{1}{2} d(\mathrm{r}) \sum_{n} \sum_{\nu} A_{n}{ }^{(\omega / 2)} A_{\nu}^{(\omega / 2)} \mathcal{E}_{\nu}{ }^{(n, \omega / 2)} \mathcal{E}_{\nu}{ }^{(p, \omega / 2)} \\
& \times e^{i\left[\omega t-\left\langle\beta_{n} \omega / 2+\beta_{p}^{\omega / \alpha}\right) z\right]}+\text { c.c. }
\end{aligned}
$$

We consider a case of a single mode input, say $n$. In that case the double summation of (36) collapses to a single term $n=p$. 
If we then use $P_{y}(\mathbf{r}, t)$ as $\left[P_{\text {pert }}(\mathbf{r}, t)\right]_{y}$ in (32) we get

$\frac{d A_{m}{ }^{(\omega)}}{d z}=-\frac{i \omega d(z)}{4}\left[A_{n}{ }^{(\omega / 2)}\right]^{2} e^{-i\left(2 \beta_{n} \omega / 2-\beta_{m} \omega\right) z} S^{(n, n, m)}$

with

$$
S^{(n, n, m)} \equiv \int_{-\infty}^{\infty} \mathcal{E}_{\nu}{ }^{(n, \omega / 2)} \mathcal{E}_{\nu}{ }^{(n, \omega / 2)} \mathcal{E}_{\nu}{ }^{(m, \omega)} f(x) d x
$$

where we took $d(\mathbf{r}) \equiv d(z) f(x)$.

In the interest of conciseness let us consider the case where the inner layer 2 is nonlinear and where both the input and output modes are well confined. We thus have $q_{m}, p_{m} \gg h_{m}$ and $h_{m} d \simeq \pi$. From (17) and (21) we get

$$
\mathcal{E}_{\nu}^{(m, \omega)} \rightarrow 2 \sqrt{\frac{\omega \mu}{\beta_{m} t}} \sin \frac{m \pi x}{t}, \quad-t \leq x \leq 0 .
$$

The overlap integral $S^{(n, n, m)}$ is maximum for $n=m=1$, i.e., fundamental mode operation both at $\omega$ and $\omega / 2$. For this case the overlap integral becomes

$$
\begin{aligned}
S^{(1,1,1)} & =\int_{-\infty}^{\infty} \mathcal{E}_{\nu}{ }^{(1, \omega / 2)} \mathcal{E}_{\nu}{ }^{(1, \omega / 2)} \mathcal{E}_{\nu}{ }^{(1, \omega)} f(x) d x \\
& =\int_{-t}^{0} \mathcal{E}_{\nu}{ }^{(1, \omega / 2)} \mathcal{E}_{\nu}{ }^{(1, \omega / 2)} \mathcal{E}_{\nu}{ }^{(1, \omega)} d x \\
& =\frac{1.2 \sqrt{2}}{\sqrt{t}} \frac{(\omega \mu)^{3 / 2}}{\left(\beta^{\omega}\right)^{1 / 2} \beta^{\omega / 2}}
\end{aligned}
$$

and (37) can be written as

$\frac{d A^{(\omega)}}{d z}=-i \frac{2^{1 / 2} \times 1.2}{4} \frac{d}{\sqrt{t}} \frac{\mu^{3 / 2} \omega^{5 / 2}}{\left(\beta^{\omega}\right)^{1 / 2} \beta^{\omega / 2}}\left(A^{(\omega / 2)}\right)^{2} e^{-i \Delta z}$

with

$$
\Delta \equiv \beta^{\omega}-2 \beta^{\omega / 2}
$$

and where the, now-superfluous, mode-number subscripts have been dropped. Integrating (40) over the interaction distance $l$ gives

$$
\left|A^{(\omega)}(l)\right|^{2}=\frac{\omega^{5} \mu^{3}(1.2)^{2} d^{2}}{8 \beta^{\omega}\left(\beta^{\omega / 2}\right)^{2} t}\left|A^{\omega / 2}\right|^{4} l^{2} \frac{\sin ^{2}(\Delta l / 2)}{(\Delta l / 2)^{2}} .
$$

The normalization condition (20) was chosen so that $|A|^{2}$ is the power per unit width in the mode. We can thus rewrite (42) as

$$
\frac{P^{\omega}}{P^{\omega / 2}}=0.72\left(\frac{\mu}{\epsilon_{0}}\right)^{3 / 2} \frac{\omega^{2} d^{2} l^{2}}{n^{3}}\left(\frac{P^{\omega / 2}}{w t}\right) \frac{\sin ^{2}(\Delta l / 2)}{(\Delta l / 2)^{2}}
$$

where we used $\beta^{\omega} \simeq \omega \sqrt{\mu \epsilon}, \epsilon / \epsilon_{0}=n^{2}$. Note that $\left(P^{\omega / 2} / w t\right)$ is the intensity (watts/square meter) of theinput mode. Except for a numerical factor of 1.44 , this expression is similar to that derived for the bulk-crystal case [13]. Efficient conver-

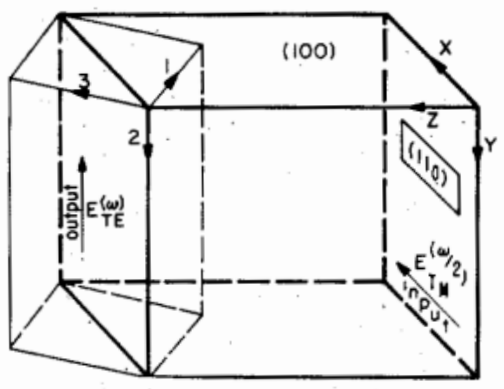

Fig. 4. The orientation of a $43 \mathrm{~m}$ crystal for converting a TM input at $\omega / 2$ to a TE wave at $\omega . x, y, z$ are the dielectric-waveguide coordinates, while 1,2 , and 3 are the crystalline axes. Top surface is (100):

sion results when the phase-matching condition

$$
\Delta \equiv \beta^{\omega}-2 \beta^{\omega / 2}=0
$$

is satisfied. In this case the factor $\sin ^{2}(\Delta l / 2)(\Delta l / 2)^{2}$ is unity. Phase-matching techniques will be discussed later.

\section{B. Case II: $T M_{\text {input }}-T E_{\text {out } p u t}$}

The anisotropy of the nonlinear optical properties can be used in such a way that the output at $\omega$ is polarized orthogonally to the field of the input mode at $\omega / 2$. To be specific, we consider the case of an input TM mode and an output TE mode. If, as an example, the guiding layer (or one of the bounding layers) belongs to the $43 \mathrm{~m}$ crystal class (GaAs, CdTe, InAs), it is possible to have a guide geometry as shown in Fig. 4. $x, y, z$ is the waveguide coordinate system as defined in Fig. 4, while 1, 2, and 3 are the conventional crystalline axes. For input TM mode with $E \| x$ we have

$$
E_{3}=E_{1}=\frac{E_{z}}{\sqrt{2}} .
$$

The nonlinear optical properties of $43 \mathrm{~m}$ crystals are described by [13]

$$
\begin{aligned}
& P_{1}=2 d_{123} E_{2} E_{3} \\
& P_{2}=2 d_{123} E_{1} E_{3} \\
& P_{3}=2 d_{123} E_{1} E_{2}
\end{aligned}
$$

so that

$$
P_{y}=P_{2}=d_{123} E_{x}^{2} .
$$

Taking

$$
H_{\nu}=\frac{1}{2} \sum_{l} B_{l} \mathcal{F C}_{\nu}{ }^{(l)}(x) e^{i\left((\omega t / 2)-\beta \omega / \alpha_{z}\right)}+\text { c.c. }
$$

and using $\left(\partial H_{y} / \partial z\right)=-i \omega \epsilon E_{x}$ gives

$$
E_{x}^{(\omega / 2)}(\mathbf{r}, t)=\frac{1}{2}\left(\frac{1}{\omega \epsilon / 2}\right) \sum_{l} \beta_{l} B_{l} \mathscr{F C}_{\nu}{ }^{(l)} e^{i\left((\omega t / 2)-\beta^{\left.\omega / z_{z}\right)}\right.}+\text { c.c. }
$$


Using (45) and assuming a single, say $m$, mode input at $\omega / 2$ results in

$$
\begin{array}{r}
P_{\nu}(\mathrm{r}, t)=\frac{d}{2}\left[\left(\frac{\beta_{m}{ }^{\omega / 2}}{\omega \epsilon / 2}\right) B_{m}{ }^{(\omega / 2)} \mathcal{C}_{\nu}{ }^{(m, \omega / 2)}(x)\right]^{2} \\
\cdot e^{i\left(\omega t-2 \beta_{m} \omega / \alpha_{z}\right)}+\text { c.c. }
\end{array}
$$

Substituting (47) into (32) we obtain

$\frac{d A_{n}^{(\omega)}}{d z}=-\frac{i \omega d}{4} \frac{\left(\beta_{m}{ }^{\omega / 2}\right)^{2}}{\omega^{2} \epsilon^{2}}\left(B_{m}{ }^{(\omega / 2)}\right)^{2} e^{i \Delta z} S^{(m, m, n)}+$ c.c.

where

$$
S^{(m, m, n)}=\int_{-\infty}^{\infty} \mathcal{H}_{y}{ }^{(m, \omega / 2)} \mathfrak{H}_{y}{ }^{(m, \omega / 2)} \mathcal{E}_{y}^{(n, \omega)} f(x) d x
$$

and

$$
\Delta=\left(\beta_{n}{ }^{\omega}\right)_{\mathrm{TE}}-2\left(\beta_{m}{ }^{\omega / 2}\right)_{\mathrm{TM}} .
$$

For the special case $m=n=1$ and for well-confined modes we have, using (17) and (22),

$$
S^{(1,1,1)}=\left(\frac{\omega \epsilon}{\beta_{\mathrm{TM}} \omega / 2}\right)\left(\frac{2 \omega \mu}{\beta_{\mathrm{TE}}{ }^{\omega}}\right)^{1 / 2} \frac{1.2}{\sqrt{t}} .
$$

Proceeding as in the previous section leads finally to

$$
\frac{\left(P^{\omega}\right)_{\mathrm{TE}}}{\left(P^{\omega / 2}\right)_{\mathrm{TM}}}=0.72\left(\frac{\mu}{\epsilon_{0}}\right)^{3 / 2} \frac{\omega^{2} d^{2} l^{2}}{n^{3}}\left(\frac{P^{\omega / 2}}{w t}\right) \frac{\sin ^{2} \Delta l / 2}{(\Delta l / 2)^{2}}
$$

an expression identical to that obtained in (43) for TE-TE conversion. We must recall, however, that the nonlinear coefficient $d$ in (51) is not necessarily the same as that appearing in (43), reflecting the differences in crystalline orientation needed to achieve coupling in each case.

\section{Phase Matching}

It follows from (43) or (51) that a necessary condition for second-harmonic generation is $\Delta l / 2 \ll \pi$ so that the factor $\sin ^{2}(\Delta l / 2) /(\Delta l / 2)^{2}$ is near unity. In this case the conversion efficiency is proportional to $l^{2}$. This phase-matching condition can be satisfied by using the dependence of the propagation constants $\beta$ of the various modes on the waveguide dimensions [7]. An alternate approach is to introduce a space-periodic perturbation into the waveguide with a period $\Lambda$ satisfying

$$
\Lambda=\frac{2 \pi}{\Delta} q, \quad q=1,2,3 \cdots
$$

Schemes based on waveguide corrugation and on modulating the nonlinear coefficient $d$ have been proposed [14]. In this section we will consider the case of $d$ modulation. We go back to (37) but allow explicitly for a spatial modula- tion of $d$ by taking $d(z)$ as

$$
d(z)=\frac{d}{2}+\sum_{q \text { odd integer }} \frac{2 d}{q \pi} \sin \frac{2 \pi q}{\Lambda} z
$$

corresponding to a square-wave alternation between 0 and $d$ with a period $\Lambda$. Instead of (37) we now have

$$
\begin{aligned}
\frac{d A_{m}{ }^{(\omega)}}{d z}=-\frac{i \omega}{4} & {\left[\frac{d}{2}-\sum_{q \text { odd }} \frac{i d}{q \pi}\left(e^{i 2 \pi \eta z / A}-e^{-i 2 \pi d z / A}\right)\right] } \\
& \times\left[A_{n}^{(\omega / 2)}\right]^{2} e^{-i\left(2 \beta_{x} \omega / \Delta-\beta_{m} \omega\right) z} S^{(n, n, m)} .
\end{aligned}
$$

We can choose the period $\Lambda$ such that for some value of $q$

$$
-\frac{2 \pi q}{\Lambda}+\beta_{m}{ }^{\omega}-2 \beta_{n}{ }^{\omega / 2}=0 .
$$

This results in a synchronous term (i.e., one with a zero exponent) on the right side of (54) so that

$$
\frac{d A_{m}{ }^{(\omega)}}{d z}=\frac{\omega d}{4 q \pi}\left[A_{n}^{(\omega / 2)}\right]^{2} S^{(n, n, m)}
$$

where the nonsynchronous terms have been neglected. A comparison to (37) shows that the effective nonlinear coefficient is now reduced to

$$
d_{\mathrm{eff}}=\frac{d}{q \pi}
$$

and that instead of (43)

$$
\frac{P^{\omega}}{P^{\omega / 2}}=0.72\left(\frac{\mu}{\epsilon_{0}}\right)^{3 / 2} \frac{\omega^{2} d_{\mathrm{eft}}^{2} l^{2}}{n^{3}}\left(\frac{P^{\omega / 2}}{\omega t}\right)
$$

operation based on $q=1$ is thus most efficient, leading to a reduction by a factor of $\pi^{2}$ in the conversion efficiency. We note, however, that the factor $\sin ^{2}(\Delta l / 2) /(\Delta l / 2)^{2}$ is now unity, which makes it possible to take advantage of the $l^{2}$ dependence of the conversion efficiency.

\section{Electrooptic Mode Coupling}

The electrooptic effect in thin-film configurations can be used in a variety of switching applications. Its use as a polarization switch in a GaAs waveguide at $1.15 \mu$ has been demonstrated [6]. In contrast to the conventional bulk [15] treatment of the electrooptic effect which relies heavily on the concept of induced retardation, we view the process as that of coupling between TE and TM modes brought about by the applied low-frequency electric field.

The linear-electrooptic effect is conventionally defined [16] in terms of a third-rank tensor $r_{i j k}$ which relates the changes in the constants of the index ellipsoid to the applied field according to

$$
\Delta\left(\frac{1}{n^{2}}\right)_{i j}=r_{i j k} E_{k}
$$


It follows from (58) that an alternative and equivalent definition would be to specify the changes of the dielectric tensor $\epsilon_{i j}$ as

$$
\Delta \epsilon_{i i}=\frac{\epsilon_{i} \epsilon_{i}}{\epsilon_{0}} r_{i j k} E_{k}^{(0)}
$$

where the (0) superscript denotes a "low" frequency, i.e., a frequency well below the crystal's Reststrahl band. Using the relations

$$
\begin{aligned}
& \mathbf{D}=\epsilon_{0} \mathbf{E}+\mathbf{P} \\
& D_{i}=\epsilon_{i j} E_{j}
\end{aligned}
$$

and choosing a principal coordinate system so that

$$
\epsilon_{i j}\left(E^{(0)} \neq 0\right)=\epsilon_{i j} \delta_{i j}+\Delta \epsilon_{i j}
$$

leads to

$$
P_{i}^{(\omega)}=\left[\frac{\epsilon_{i} \epsilon_{j}}{\epsilon_{0}} r_{i j k} E_{k}^{(0)}+\left(\epsilon_{i j}-\epsilon_{0}\right) \delta_{i j}\right] E_{j}^{(\omega)}
$$

where we used the convention $\epsilon_{i} \equiv \epsilon_{i i}$. The perturbation polarization to be used in (32) is that part of $P_{i}^{(\omega)}$ which is proportional to the "low"-frequency electric field, i.e.,

$\left[P_{\text {Dert }}(\mathbf{r}, t)\right]_{i}=\frac{\epsilon_{i} \epsilon_{j} r_{i j k}}{\epsilon_{0}} E_{k}{ }^{(0)}\left[\frac{E_{j}{ }^{(\omega)}}{2}-e^{i(\omega t-\beta z)}+\right.$ c.c. $]$.

To be specific, we assume that the input is a TM mode with $\mathbf{E}^{(\omega)} \| \mathbf{a}_{x}$ which is coupled by the electrooptic properties of the bounding media or the guiding layer to the TE mode with $\dot{\mathbf{E}}^{(\boldsymbol{\omega})} \| \mathbf{a}_{y}{ }^{2}$ The starting point is again (32) where the mode $m$ corresponds to the output TEand $\left[P_{\text {pert }}\right]_{y}$ is the $y$ component of the polarization (61) induced by the $x$ (and $z^{*}$ ) electricfield components of the input TM mode. Using (61) we get

$$
P_{\nu}{ }^{(\omega)}=\frac{\epsilon_{i} \epsilon_{i} r_{i j k} l_{i j} l_{i x} E_{k}^{(0)}}{\epsilon_{0}} E_{x}^{(\omega)}
$$

where the l's are direction cosines. Defining

$$
\epsilon_{i} \epsilon_{j} r_{i j k} l_{i y} l_{j x} E_{k}^{(0)} \equiv \epsilon^{2} r E^{(0)}
$$

(62) becomes

$$
P_{\nu}^{(\omega)}=\frac{\epsilon^{2} r E^{(0)}}{\epsilon_{0}} E_{x}^{(\omega)}
$$

where $P_{y}{ }^{(\omega)}$ is the complex amplitude of the polarization. In most cases of practical interest the choice of crystal orientation and the field $E_{k}^{(0)}$ is such as to simplify (63) to a simple form resembling (64); an example is provided at the end of Section VI. In any case, the definition of (63) applies to the most general case.

\footnotetext{
${ }^{2}$ The $E_{z}$ component of a TM mode can also cause coupling but this will typically be a smaller effect, since $E_{z} \ll E_{x}$.
}

Using (22) the $E_{x}$ component of a single forwardtraveling TM mode is given by

$$
E_{x}^{(l)}(\mathbf{r}, t)=\frac{\beta_{l}}{2 \omega \epsilon(x)} B_{l} \mathcal{H}_{y}^{(l)}(x) e^{i\left(\omega t-\beta_{l z}\right)}
$$

where the normalization (26) is such that $\left|B_{l}\right|^{2}$ is the power per unit width in the mode. From (64) and (65) we obtain

$P_{\nu}(\mathbf{r}, t)=\frac{\epsilon^{2} r(x, z) E^{(0)}}{2 \omega \epsilon_{0} \epsilon(x)} \beta_{l} B_{l} \mathcal{H}_{\nu}{ }^{(l)}(x) e^{i\left(\omega t-\beta_{l z}\right)}+$ c.c.

Substitution of (66) into the wave equation (32) leads to

$$
\begin{aligned}
& \frac{d A_{m}{ }^{+}}{d z} \exp \left(-i \beta_{m}{ }^{\mathrm{TL}} z\right)-\frac{d A_{m}{ }^{-}}{d z} \exp \left(i \beta_{m}{ }^{\mathrm{TH}} z\right) \\
& =-\frac{i}{4} \int_{-\infty}^{\infty} \frac{\epsilon^{2} r(x, z) E^{(6)}(x, z)}{\epsilon(x) \epsilon_{0}} \beta_{l} B_{l} \mathcal{H}{ }^{(t)}(x) \mathcal{E}_{y}{ }^{(m)}(x) d x \\
& \quad \times \exp \left(-i \beta_{l}{ }^{\mathrm{TM}} z\right)+\text { c.c. }
\end{aligned}
$$

Equation (67) is general enough to apply to a large variety of cases. The dependence of $E^{(0)}$ and $r(x, z)$ on $x$ allows for coupling by electrooptic material in the guiding or in the bounding layers. The $z$ dependence allows for situations where $E^{(0)}$ or $r$ depend on position. To be specific, we consider first the case where the guiding layer $-t<x<0$ is uniformly electrooptic and where $E^{(0)}$ is uniform over the same region, so that the integration in (67) is from $-t$ to 0 . In that case, the overlap integral of (67) is maximum when the TE $(m)$ and $\mathrm{TM}(l)$ modes are well confined and of the same order so that $l=m$. Under well-confined conditions $p, q \gg h$ and the expressions (17) for $\varepsilon_{y}{ }^{(m)}(x)$ and (24) for

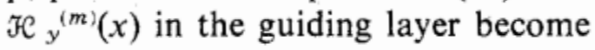

$$
\begin{gathered}
\mathcal{E}_{y}{ }^{(m)}(x) \rightarrow\left(\frac{4 \omega \mu}{t \beta_{m}{ }^{\mathrm{TE}}}\right)^{1 / 2} \sin \frac{m \pi x}{t} \\
\mathfrak{F}_{y}{ }^{(m)}(x) \rightarrow\left(\frac{4 \omega \epsilon_{0} n_{2}{ }^{2}}{t \beta_{m}{ }^{\mathrm{TM}}}\right)^{1 / 2} \sin \frac{m \pi x}{t}
\end{gathered}
$$

where for well-confined mode $\beta_{l}^{\mathrm{TM}} \simeq \beta_{m}{ }^{\mathrm{TE}} \equiv \beta=k n_{2}$. In this case the overlap integral becomes

$$
\int_{-t}^{0} \mathcal{F}_{y}{ }^{(m)}(x) \mathcal{E}_{y}{ }^{(m)}(x) d x=\frac{4 \omega \sqrt{\mu \epsilon_{2}}}{t \beta} \int_{-t}^{0} \sin ^{2} \frac{m \pi x}{t} d x=2 .
$$

Having chosen the case of a uniform $E^{(0)}$ and $r$, the only $z$ dependence on the right side of (67) is that of the $\exp \left(-i \beta_{l}{ }^{\mathrm{TM}} z\right)$ factor. Since $\beta_{m}{ }^{\mathrm{TM}} \simeq \beta_{m}{ }^{\mathrm{TE}}(l=m)$ we may neglect the term involving $A_{m}{ }^{-}$. The coupling thus involves only the forward TE and TM modes. Using (69), (67) becomes

$$
\frac{d A_{m}}{d z}=-i_{K} B_{m} \exp \left[-i\left(\beta_{m}{ }^{\mathrm{TM}}-\beta_{m}{ }^{\mathrm{Tl}}\right) z\right]
$$


while from (4)

$$
\begin{gathered}
\frac{d B_{m}}{d z}=-i \kappa A_{m} \exp \left[i\left(\beta_{m}{ }^{\mathrm{TM}}-\beta_{m}{ }^{\mathrm{TE}}\right) z\right] \\
\kappa=\frac{n_{2}{ }^{3} k r E^{(0)}}{2} .
\end{gathered}
$$

The form of (70) will apply to the general case involving arbitrary spatial dependence of $r$ and $E^{(0)}$. In that case we need to perform the integration in (67) to evaluate the coupling coefficient $\kappa$.

The form of (70) is identical to that of (2). The solution of $(70)$ is thus given by (6) with

$$
\Delta=\beta_{m}{ }^{\mathrm{TM}}-\beta_{m}^{\mathrm{TE}} .
$$

The transfer of power between the modes for the phasematched $(\Delta=0)$ and $\Delta \neq 0$ case are as shown in Fig. 1. A complete transfer of power between the modes thus requires that $\Delta=0$, i.e., phase matching. Means for phase matching will be discussed in Section VI. For the meantime let us assume that $\kappa \gg \Delta$ so that, according to (6), the effects of phase mismatch can be neglected. A complete power transfer in this case occurs in a distance $l$ such that

$$
\kappa l=\pi / 2
$$

or using (71)

$$
l E=\frac{\lambda_{0}}{2 n_{2}{ }^{3} r}
$$

where $\lambda_{0}=2 \pi / k$. The product $l E$ is identical to the "halfwave" voltage of bulk electrooptic modulators [15]. The "half-voltage" in the bulk case, we recall, is the fieldlength product which causes a $90^{\circ}$ rotation in the plane of polarization of a wave incident on an electrooptic crystal.

Unlike the bulk case, the coupling between the two guided modes can take place even when the electrooptic perturbation is limited to an arbitrarily small portion of the transverse dimensions [6] or when the two modes are of different order $(l \neq m)$.

To appreciate the order of magnitude of the coupling, consider a case where the guiding layer is GaAs and $\lambda_{0}=1$ $\mu \mathrm{m}$. In this case [15]

$$
n_{2} \simeq 3.5, \quad n_{2}{ }^{3} r=59 \times 10^{-12} \frac{\mathrm{m}}{\mathrm{V}} .
$$

Taking an applied field $E^{(0)}=10^{8} \mathrm{~V} / \mathrm{m}$ we obtain from (71)

$$
\begin{aligned}
& \kappa=1.85 \mathrm{~cm}^{-1} \\
& l \equiv \frac{\pi}{2 \kappa}=0.85 \mathrm{~cm}
\end{aligned}
$$

for the coupling constant and the power-exchange distance, respectively.

\section{Phase Matching in Electrooptic Coupling}

In general, $\beta^{\mathrm{TM}} \neq \beta^{\mathrm{TE}}$ even for the same-order mode so that the fraction of the power exchanged in the electrooptic-coupling case described previously does not exceed, according to (6), $\kappa^{2} /\left(\kappa^{2}+\Delta^{2}\right)$. If $\Delta \gg \kappa$, the coupling is negligible. To appreciate the importance of this fact, let us use the numerical data of the example considered at the end of Section V. We have $k=1.85 \mathrm{~cm}^{-1}$ and $\beta \simeq n_{2} k$ $\simeq 2.2 \times 10^{5} \mathrm{~cm}^{-1}$. The exchange factor $\kappa^{2} /\left(\kappa^{2}+\Delta^{2}\right)$ is thus reduced to 0.5 when $\Delta / \beta \simeq\left[\left(B_{\mathrm{TE}}-B_{\mathrm{TM}}\right) / B_{\mathrm{TE}}\right] \sim 10^{-5}$. The critical importance of phase matching is thus manifest. Since the dispersion due to the waveguide will in general be such as to make $\Delta \gg \kappa$, some means for phase matching are necessary. We start by considering again the coupled-mode equations (70), reintroducing the possible $z$ dependence of $\kappa$

$$
\begin{aligned}
\frac{d A_{m}}{d z} & =-i \kappa(z) B_{m} e^{+i \Delta z} \\
\frac{d B_{m}}{d z} & =-i \kappa(z) A_{m} e^{-i \Delta z} \\
\dot{\Delta} & \equiv \beta_{m}{ }^{\mathrm{TE}}-\beta_{m}{ }^{\mathrm{TM}}
\end{aligned}
$$

with

$$
\kappa(z)=n_{2}{ }^{3} k r(z) E^{(0)}(z) .
$$

As in the case of second-harmonic generation, we can use a spatial modulation of $r$ or the field $E^{(0)}$ for phase matching. Consider, for example, the case where the field $E^{[0]}(z)$ reverses its direction periodically as with the electrode arrangement of Fig. 5. Approximating the electric field in the guiding layer by

$$
E^{(0)}(z)=\sum_{\text {odd }} \frac{4 E_{0}}{q \pi} \sin \frac{2 q \pi}{\Lambda} z
$$

corresponding to a field reversal between $E_{0}$ and $-E_{0}$ every $\Lambda$ meters, we can take $\kappa(z)$ in (74) as

$$
\begin{aligned}
\kappa(z) & =-i \kappa_{0} \sum_{q} \frac{2}{q \pi}\left(e^{(i 2 \pi q / \Lambda) z}-e^{-(i 2 \pi q / \Lambda) z}\right) \\
\kappa_{0} & =n_{2}{ }^{3} k r E_{0} .
\end{aligned}
$$

If we substitute (76) in (74) we obtain on the right-side terms with exponential dependence of the type

$$
\exp i\left( \pm \Delta \pm \frac{2 q \pi}{\Lambda}\right) z
$$

One can choose $\Lambda$ such that, for some $q,(2 \pi q / \Lambda)=\Delta$. This results in a synchronous driving term (i.e., one with a zero ex- 


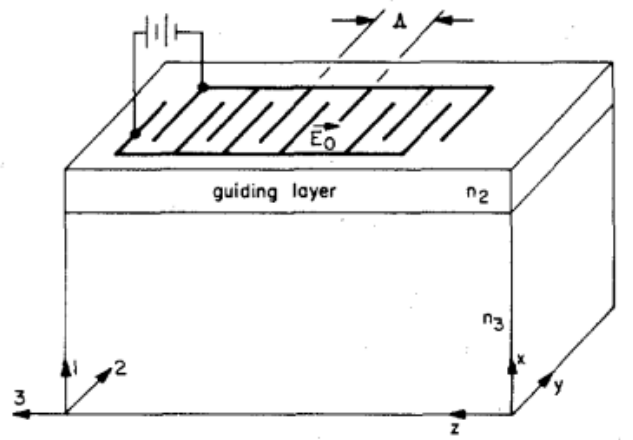

Fig. 5. An interdigital-electrode structure for applying a spatially modulated electric field in electrooptic phase matching. $x, y$, and $z$ are the waveguide coordinates, while 1,2 , and 3 refer to the cubic [100] axes of a $43 \mathrm{~m}$ crystal.

ponent). To be specific, let us choose

$$
\frac{2 \pi}{\Lambda}=\Delta
$$

and keeping only the synchronous term, obtain from (74)

$$
\begin{aligned}
& \frac{d A_{m}}{d z}=\frac{\kappa_{0}}{(\pi / 2)} B_{m} \\
& \frac{d B_{m}}{d z}=-\frac{\kappa_{0}}{(\pi / 2)} A_{m} .
\end{aligned}
$$

This corresponds to phase-matched operation with an effective coupling coefficient reduced by $\pi / 2$ relative to phasematched operation with a uniform field $E^{(0)}(z)=E_{0}$. The solution of (78) is given by (7).

We close this section by considering, again, the use of $43 \mathrm{~m}$ crystals for the phase-matching scheme just discussed. The nonvanishing elements of the $r_{i j k}$ tensor are [15] $r_{321}=r_{312}=$ $r_{123}$. From (61) it follows directly that a $43 \mathrm{~m}$ crystal oriented, as in Fig. 5, so that its cubic 1, 2, 3 axes coincide, respectively, with the $x, y, z$ directions of the waveguide, is optimal sincein this case

$$
P_{x}{ }^{(\omega)}=\frac{\epsilon^{2}}{\epsilon_{0}} r_{123} E_{z}{ }^{(0)} E_{\nu}{ }^{(\omega)} \quad P_{\nu}{ }^{(\omega)}=\frac{\epsilon^{2}}{\epsilon_{0}} r_{123} E_{z}{ }^{(0)} E_{z}{ }^{(\omega)}
$$

thus coupling the TE mode $\left(E_{y}{ }^{(\omega)}\right)$ to the $\mathrm{TM}\left(E_{x}{ }^{(\omega)}\right)$, and vice versa, in the presence of a longitudinal dc field $E_{z}{ }^{\left({ }^{0}\right)}$.

\section{Photoelastic Coupling}

The possibility of coupling dielectric-waveguide optical modes through the intermediary of sound waves has been demonstrated [17]. In this section we will treat this class of interactions using the coupled-mode formalism.

The photoelastic effect is defined by relating the effect of strain $S_{k l}$ on the constants of the index ellipsoid through [18]

$$
\Delta\left(\frac{1}{n^{2}}\right)_{i j}=p_{i j k l} S_{k l} .
$$

$P_{i j k l}$ is the photoelastic tensor. Comparing (79) to (58) we can apply the results of Section $\mathrm{V}$ directly. Taking the strain field in the form of

$$
S_{k l}{ }^{(\Omega)}(\mathrm{r}, t)=\frac{1}{2} S_{k l}{ }^{(\Omega)} e^{i(\Omega t-K z)}+\text { c.c. }
$$

we obtain in a manner similar to (61)

$$
\begin{aligned}
{\left[P_{\mathrm{pert}}(\mathrm{r}, t)\right]_{i} } & =\frac{\epsilon_{i} \epsilon_{j} p_{i i k l}}{4 \epsilon_{0}}\left[S_{k l}{ }^{(\Omega)} E_{j}^{(\omega)} e^{i((\omega+\Omega) t-(\beta+K) z]}\right. \\
& \left.+S_{k l}{ }^{(-\Omega)} E_{j}{ }^{(\omega)} e^{i[(\omega-\Omega) t-(\beta-K) z]}\right]+ \text { c.c. }
\end{aligned}
$$

for the polarization wave arising from the nonlinear mixing of an electric field

$$
\frac{1}{2} E_{j}^{(\omega)} e^{i(\omega t-\beta z)}+\text { c.c. }
$$

and a sound strain wave (80).

To be specific, we will assume again that the input optical field is a TM mode and will derive the equation governing the evolution of the TE mode due to the coupling. In a manner similar to (63) we abbreviate the information relating to crystal symmetry and orientation by defining

$$
\epsilon^{2} p S \equiv \epsilon_{i} \epsilon_{j} p_{i j k l} S_{k l} l_{j k} l_{i y}
$$

and instead of (81) use

$$
\begin{aligned}
& {\left[P_{\text {pert }}(\mathrm{r}, t)\right]_{y}} \\
& \quad=\frac{\epsilon^{2}}{4 \epsilon_{0}} p\left[S^{(\Omega)} E_{x}^{(\omega)} \exp \left\{i\left[(\omega+\Omega) t-\left(\beta_{\mathrm{TM}}+K\right) z\right]\right\}\right. \\
& \left.\quad+S^{(-\Omega)} E_{x}^{(\omega)} \exp \left\{i\left[(\omega-\Omega) t-\left(\beta_{\mathrm{TM}}-K\right) z\right]\right\}\right]+ \text { c.c. }
\end{aligned}
$$

In a manner identical to that leading to (67) we obtain

$$
\begin{aligned}
& \frac{d A_{m}{ }^{(+)}}{d z} \exp \left[i\left(\omega_{\mathrm{TE}} t-\beta_{\mathrm{TE}} z\right)\right] \\
& \quad-\frac{d A_{m}{ }^{(-)}}{d z} \exp \left[i\left(\omega_{\mathrm{TE}} t+\beta_{\mathrm{TE}} z\right)\right] \\
& =-\frac{i}{8} \int_{-\infty}^{\infty} \frac{\epsilon^{2} p(x, z) S^{\Omega}(x)}{\epsilon(x) \epsilon_{0}} \beta_{l} B_{l} \mathcal{H}_{\nu}{ }^{(l)}(x) \mathcal{E}_{\nu}{ }^{(m)}(x) d x \\
& \quad \cdot\left[\exp \left\{i\left[(\omega+\Omega) t-\left(\beta_{\mathrm{TM}}+K\right) z\right]\right\}\right. \\
& \left.+\exp \left\{i\left[(\omega-\Omega) t-\left(\beta_{\mathrm{TM}}-K\right) z\right]\right\}\right] .
\end{aligned}
$$

A few comments may be in order here. Each of the two terms on the right-hand side of (84) represents a traveling polarization wave. Both input waves, i.e., $S^{(\Omega)}$ and $E_{x}^{(\omega)}$, we recall, are taken as traveling in the $+z$ direction. Ordinarily, $\beta_{\mathrm{TE}}$ is close to, but slightly larger than, $\boldsymbol{\beta}_{\mathrm{TM}}$. In this case the coupling is via the first term on the right side of (84) and the wavelength of the sound wave is adjusted so that

$$
\beta_{\mathrm{TB}}=\beta_{\mathrm{TM}}+K
$$


and the resulting TE mode is shifted up in frequency to ${ }^{3}$

$$
\omega_{\mathrm{TE}}=\omega+\Omega .
$$

Since the sign of $\beta_{\mathrm{TE}}$ and $\beta_{\mathrm{TM}}$ is the same, the coupling is codirectional. This is the case which we consider in detail below. Since $K / \beta=\left(c / v_{s}\right)(\Omega / \omega)$, where $v_{s}$ is the sound velocity, it is possible for reasonable values of the sound frequency $\Omega$ to have $K \simeq 2 \beta$. In this case the second term on the right side of (84) represents a polarization wave traveling in the $-z$ direction with a phase velocity $-\omega /(K$ $-\beta) \simeq(-\omega / \beta)$. This wave is capable of coupling to the backward TE (or TM) mode. In this case we have

$$
\begin{aligned}
& \beta_{\mathrm{TE}}=B_{\mathrm{TM}}-K<0 \\
& \omega_{\mathrm{TE}}=\omega+\Omega .
\end{aligned}
$$

Another possibility exists when the sound wave travels oppositely to the input TM mode. In this case we merely reverse the sign of $K$ in (84). Codirectional coupling is now provided by the second term on the right side of (84) with

$$
\begin{aligned}
& \beta_{\mathrm{TE}}=\beta_{\mathrm{TM}}+K \\
& \omega_{\mathrm{TE}}=\omega-\Omega
\end{aligned}
$$

where the fact that now $\omega_{T E}<\omega$ can be understood by noting that for each photon removed by the interaction from the input TM mode one new (negative traveling) phonon and one new TE photon are generated. Contradirectional coupling can take place due to the first term when

$$
\begin{aligned}
& \beta_{T E}=\beta_{T M}-K<0 \\
& \omega_{T E}=\omega+\Omega .
\end{aligned}
$$

Returning to the codirectional-coupling case represented by (85), we obtain, following the same steps leading to (70),

$$
\begin{aligned}
d A_{m}{ }^{(\omega+\Omega)} / d z & =i \kappa B_{m}{ }^{(\omega)} e^{-i \Delta z} \\
d B_{m}{ }^{(\omega)} / d z & =-i \kappa A_{m}{ }^{(\omega+\Omega)} e^{i \Delta z} \\
\Delta & \equiv K-\left(\beta_{\mathrm{TL}}-\beta_{\mathrm{TM}}\right)
\end{aligned}
$$

where we assumed $\omega \gg \Omega$. In the case of well-confined modes and of a photoelastic medium filling uniformly the guiding region 2 , the coupling constant, following the procedure leading to (71), is found to be

$$
\kappa=\frac{\pi p S^{(n)} n_{2}{ }^{3}}{2 \lambda_{0}}
$$

\footnotetext{
${ }^{3} \mathrm{~A}$ quantum mechanical analysis of this phenomenon [19] shows that in the section of the waveguide in which the TE mode grows, phonons combine with TM photons on a one to one basis to generate TE photons so that $\omega_{\mathrm{TE}}=\omega_{\mathrm{TM}}+\Omega$.
}

which is similar to $k$ of (71) except that the photoelastic constant $p$ replaces $r$, the electrooptic constant, and a factor of 2 appears in the denominator. The latter is due to the fact that the sound strain was taken as a time-harmonic field while, in the electrooptic case, the modulation field $E^{(0)}$ was taken as a dc field. The solution of $(89)$ is given by (6) and illustrated by Fig. 1. Complete power transfer can take place only when $\Delta=0$, i.e., when

$$
K=\beta_{\mathrm{TE}}-\beta_{\mathrm{TM}} .
$$

Since $K=\Omega / v_{s}$, this condition can be fulfilled by adjusting the sound frequency $\Omega$. Under phase-matched conditions we have, according to (6)

$$
\begin{aligned}
\left|A_{m}{ }^{(\omega+\Omega)}\right|^{2} & =\left|B_{m}(0)\right|^{2} \sin ^{2} \kappa z \\
\left|B_{m}{ }^{(\omega)}\right|^{2} & =\left|B_{m}(0)\right|^{2} \cos ^{2} \kappa z
\end{aligned}
$$

with complete power exchange in a distance

$$
l=\pi / 2 \kappa
$$

It is of interest to estimate the acoustic power needed to satisfy the switching condition (93). Solving (93) for the strain using $(90)$ gives

$$
S^{2}=\frac{\lambda_{0}{ }^{2}}{l^{2} p^{2} n^{6}} .
$$

The corresponding acoustic intensity $I\left(\mathrm{~W} / \mathrm{m}^{2}\right)$ can be obtained using the relation $I=\left[\left(\rho v_{s}^{3} S^{2}\right) / 2\right]$ where $\rho$ is the mass density. The result is

$$
I_{\text {switehing }}=\frac{\lambda_{0}{ }^{2} \rho v_{s}{ }^{3}}{2 l^{2} p^{2} n^{6}}=\frac{\lambda_{0}{ }^{2}}{2 l^{2} M}
$$

where $M \equiv n^{6} p^{2} / \rho v_{s}{ }^{3}$ is the acoustic figure of merit [18].

In a GaAs crystal, as an example, using the following data: $M \simeq 10^{-13}, l=5 \mathrm{~mm}$, and an optical wavelength $\lambda_{0}$ $=1 \mu \mathrm{m}$, we get

$$
I_{\text {switching }}=20 \mathrm{~W} / \mathrm{cm}^{2} \text {. }
$$

The corresponding strain amplitude is

$$
S^{(\Omega)} \cong 2.3 \times 10^{-5}
$$

where we used

$$
\rho=5.34 \mathrm{~g} / \mathrm{cm}^{3} \text { and } v_{\mathrm{s}}=5.15 \times 10^{3} \mathrm{~m} / \mathrm{s} .
$$

\section{Coupling by a Surface Corrugation}

Consider an idealized dielectric waveguide such as that in Fig. 3. Let us next perturb the spatial distribution of $n^{2}$ slightly from that shown in the figure. If the perturbation is small it is useful to consider its effect in terms of cou- 


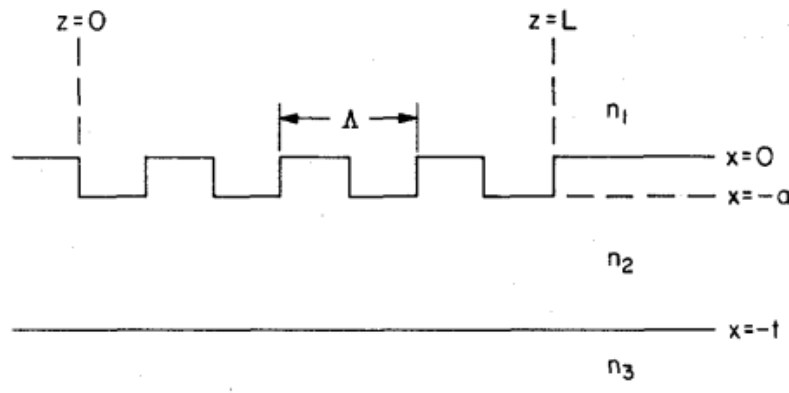

Fig. 6. A corrugated dielectric waveguide.

pling of the modes of the unperturbed system [11]. In this section we will consider a perturbation due to a mechanical corrugation of the interface as shown in Fig. 6. Using the relation

$$
\mathbf{P}=\left[\epsilon(\mathbf{r})-\epsilon_{0}\right] \mathbf{E}
$$

we get

$$
\mathbf{P}_{\text {pert }}=\Delta \epsilon(\mathbf{r}) \mathbf{E}(r, t)=\Delta n^{2}(\mathbf{r}) \epsilon_{0} \mathbf{E}(r, t)
$$

where $\epsilon(\mathbf{r})=n^{2}(\mathbf{r}) \epsilon_{0}$ so that coupling is only between TE or TM modes but not from TE to TM. To be specific, consider the case of a TE mode of order $m$ propagating in the $+z$ direction in a smooth waveguide. At $z=0$ it encounters a corrugated region, as shown in Fig. 6, extending to $z=L$.

Using (30) for $E_{y}$ and limiting the summation to a single term $l=m$ gives upon substitution in (95)

$$
\begin{array}{r}
{\left[P_{\mathrm{pert}}(\mathrm{r}, t)\right]_{\nu}=\frac{\Delta n^{2}(\mathrm{r}) \epsilon_{0}}{2}\left[A_{m}{ }^{(+)}(z) \mathcal{E}_{\nu}{ }^{(m)}(x)\right.} \\
\left.\cdot e^{i\left(\omega t-\beta_{m} z\right)}+\text { c.c. }\right] .
\end{array}
$$

We anticipate that the period $\Lambda$ will be chosen so that $2 \pi / \Lambda \simeq 2 \beta_{m}$ and the coupling will thus take place predominantly between the forward and backward modes of order $m$. Substituting (96) in (32) and limiting the left side of the latter to the backward $A_{m}^{(-)}$term gives

$$
\frac{d A_{m}{ }^{(-)}}{d z}=\frac{i \omega \epsilon_{0}}{4} A_{m}{ }^{(+)} e^{-2 i \beta_{m z} z} \int_{-\infty}^{\infty} \Delta n^{2}(x, z)\left[\mathcal{E}_{y}{ }^{(m)}(x)\right]^{2} d x .
$$

From Fig. 6 we have

$$
\begin{aligned}
\Delta n^{2}(x, z)=\Delta n^{2}(x)\left[\frac{1}{2}+\frac{2}{\pi}(\sin \eta z\right. & \left.\left.+\frac{1}{3} \sin 3 \eta z+\cdots\right)\right] \\
& \equiv \sum_{l} \Delta n_{l}{ }^{2}(x, z)
\end{aligned}
$$

where

$$
\begin{aligned}
\Delta n^{2}(x) & = \begin{cases}n_{2}{ }^{2}-n_{1}{ }^{2}, & -a \leq x \leq 0 \\
0, & \text { elsewhere }\end{cases} \\
\eta & \equiv 2 \pi / \Lambda .
\end{aligned}
$$

Coupling from $A_{m}^{(+)}$to $A_{m}^{(-)}$occurs when, for some $l$,

$$
l \eta \equiv l \frac{2 \pi}{\Lambda} \simeq 2 \beta_{m} .
$$

In this case, keeping only the synchronous term, we obtain from (97) and (98)

$$
\begin{gathered}
\frac{d A_{m}{ }^{(-)}}{d z}=\frac{\omega \epsilon_{0}}{4 \pi l} A_{m}{ }^{(+)} e^{i \Delta z} \int_{-\infty}^{\infty} \Delta n^{2}(x)\left[\mathcal{E}_{y}{ }^{(m)}(x)\right]^{2} d x \\
\Delta \equiv l \eta-2 \beta_{m} .
\end{gathered}
$$

The next task is to evaluate the integral in (100). Using (99), the integral can be written as

$$
\begin{aligned}
\int_{-\infty}^{\infty} \Delta n^{2}(x)\left[\mathcal{E}_{y}{ }^{(m)}(x)\right]^{2} d x=\left(n_{2}{ }^{2}-n_{1}{ }^{2}\right) \\
\cdot \int_{-a}^{0}\left[\mathcal{E}_{y}{ }^{(m)}(x)\right]^{2} d x=\left(n_{2}{ }^{2}-n_{1}{ }^{2}\right) C_{m}{ }^{2} \\
\cdot \int_{-a}^{0}\left[\cos \left(h_{m} x\right)-\frac{q_{m}}{h_{m}} \sin \left(h_{m} x\right)\right]^{2} d x
\end{aligned}
$$

Although the integral in (102) can be calculated exactly using (19) and (21), an especially simple result ensues if we consider that operation is sufficiently above the propagation cutoff, so that $q_{m} \gg h_{m}{ }^{4}$ Performing the integration and assuming $h a<<1$ results in

$$
\begin{aligned}
\left(n_{2}{ }^{2}-n_{1}{ }^{2}\right) \int_{-a}^{0} & {\left[\mathcal{E}_{y}{ }^{(m)}(x)\right]^{2} d x } \\
& =\frac{C_{m}{ }^{2} q_{m}{ }^{2} a^{3}}{3}\left(1+\frac{3}{q_{m} a}+\frac{3}{q_{m}{ }^{2} a^{2}}\right) .
\end{aligned}
$$

In the well-confined regime, $q_{m}, p_{m} \gg h_{m}$ so that (21) becomes

$$
C_{m}{ }^{2} \rightarrow \frac{4 h_{m}^{2} \omega \mu}{\beta_{m} t q_{m}{ }^{2}}
$$

Using $\beta_{m} \rightarrow n_{2} k, h_{m} \rightarrow \pi / t$, and (104) in (103) leads to

$$
\begin{aligned}
\left(n_{2}{ }^{2}-n_{1}{ }^{2}\right) \int_{-a}^{0}\left[\varepsilon_{y}{ }^{(m)}(x)\right]^{2} d x=\left(n_{2}{ }^{2}-\right. & \left.n_{1}{ }^{2}\right) \frac{4 \pi^{2} \omega \mu}{3 n_{2} k}\left(\frac{a}{t}\right)^{3} \\
\cdot & {\left[1+\frac{3}{q_{m} a}+\frac{3}{q_{m}{ }^{2} a^{2}}\right] }
\end{aligned}
$$

${ }^{4}$ Well above threshold $q_{m} / h_{m} \rightarrow\left(n_{2}{ }^{2}-n_{1}{ }^{2}\right)^{1 / 2}\left(2 t / \lambda_{0}\right)$. 
which upon substitution into (100) gives

$$
\frac{d A_{m}^{(-)}}{d z}=\kappa_{l} A_{m}^{(+)} e^{i \Delta z}
$$

and using (10)

$$
\begin{aligned}
\frac{d A_{m}{ }^{(+)}}{d z} & =\kappa_{l} A_{m}{ }^{(-)} e^{-i \Delta z} \\
\kappa_{l} & =\frac{\pi k}{3 l} \frac{\left(n_{2}{ }^{2}-n_{1}{ }^{2}\right)}{n_{2}}\left(\frac{a}{t}\right)^{3}\left[1+\frac{3\left(\lambda_{0} / a\right)}{2 \pi\left(n_{2}{ }^{2}-n_{1}{ }^{2}\right)^{1 / 2}}\right. \\
& \left.+\frac{3\left(\lambda_{0} / a\right)^{2}}{4 \pi^{2}\left(n_{2}{ }^{2}-n_{1}{ }^{2}\right)}\right]
\end{aligned}
$$

where $l$, we recall, is the order of the (corrugation function) harmonic responsible for the coupling, and $\lambda_{0} \equiv$ $2 \pi / k$.

The behavior of the incident and reflected waves $A_{m}^{(+)}$ and $A_{m}^{(-)}$is given by (14) and is illustrated by Fig. 2 [in which the reflected wave is $A(z)$ and the incident wave $B(z)$ ]. The exponential decay behavior occurs only for a narrow range of frequencies which satisfy, according to (13), the condition

$$
\Delta \equiv \eta-2 \beta(\omega)<2 \kappa
$$

where $\eta=2 \pi / \Lambda$ and we consider the case $l=1$ only. This behavior is formally analogous to Bragg scattering of Bloch electron waves in a crystal from one edge of the Brillouin zone to the other by the crystal periodicity [20]. The latter phenomenon is responsible for the appearance of forbidden energy gaps. The behavior of the corresponding optical gap can be elucidated by considering the total propagation constant of, say, the incident wave $B(z)$ of (12) $\left[A^{+}(z)\right.$ in the notation of this section]. From (8) and (12) we can write it as

$$
\begin{aligned}
\beta^{\prime}(\omega) & =\beta(\omega)-\frac{\Delta}{2} \pm i \frac{S}{2}=\frac{\eta}{2} \pm i \frac{S}{2} \\
S & =\sqrt{4 \kappa^{2}-\Delta^{2}} .
\end{aligned}
$$

The imaginary part of $\beta^{\prime}$ is then given by

$$
\begin{aligned}
& \operatorname{Im} \beta^{\prime}(\omega)=\sqrt{\kappa^{2}-(\beta-\eta / 2)^{2}} \\
& \simeq \sqrt{\kappa^{2}-\frac{n_{\mathrm{eff}}^{2}}{c^{2}}\left(\omega-\omega_{0}\right)^{2}}
\end{aligned}
$$

where $\omega_{0}$, the midgap frequency, is defined by $\beta\left(\omega_{0}\right)=\eta / 2$; and to get the second equality we approximated the unperturbed behavior of $\beta$ by $\beta(\omega) \simeq(\omega / c) n_{\text {eff }}$. The height of the energy gap is thus the frequency region over which $\beta^{\prime}$ is complex. Using (109) it is given by

$$
(\Delta \omega)_{\mathrm{gED}} \equiv \omega_{u}-\omega_{l}=\frac{2 \kappa c}{n_{\mathrm{eff}}}
$$

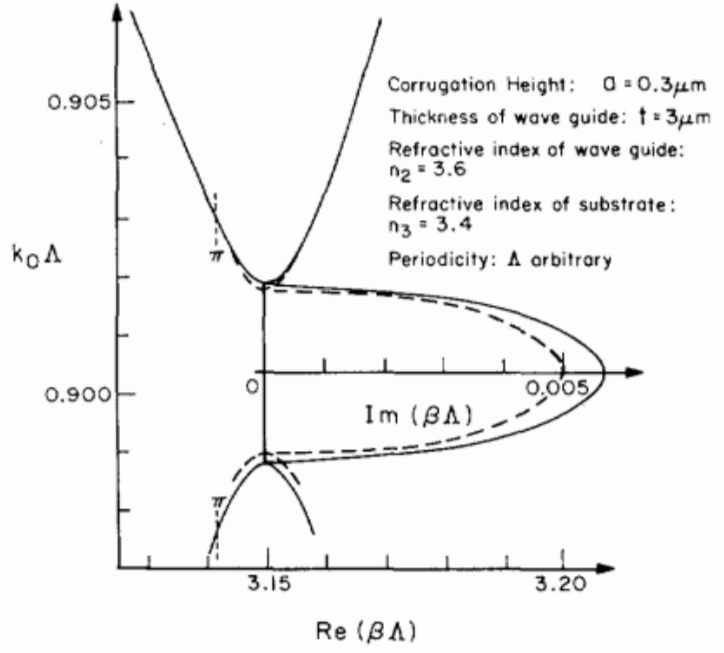

Fig. 7. A plot of the dispersion ( $k \Lambda$ versus $\beta \Lambda$ ) diagram in the vicinity of the optical gap.

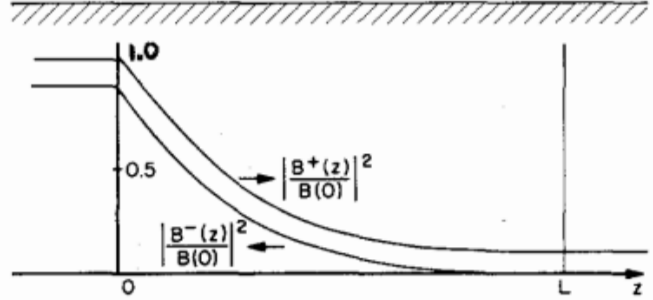

Fig. 8. A theoretical plot of the transmission characteristics (12) of a periodic waveguide near the Bragg (optical-gap) regime.

where $\omega_{u}$ and $\omega_{l}$ are the upper- and lower-gap frequencies, respectively.

The behavior of $\beta^{\prime}$ at $\omega>\omega_{u}$ and $\omega<\omega_{l}$ is likewise derivable from (108). It is given for $\omega>\omega_{u}$, as an example, by

$$
\beta^{\prime}=\frac{\eta}{2} \pm \sqrt{\frac{n_{\mathrm{eff}}^{2}}{c^{2}}\left(\omega-\omega_{u}\right)^{2}+\frac{2 n_{\mathrm{eff}} K}{c}\left(\omega-\omega_{u}\right)} .
$$

Equations (109) and (111) are valid for any coupling between the forward and backward modes of a waveguide which is describable by a set of equations such as (105), regardless of the physical mechanism responsible for the coupling. As an illustration of the above ideas we chose to plot the $\omega-\beta^{\prime}$ diagram in the vicinity of the optical gap using the case of coupling by a surface corrugation. Referring to Fig. 7 we used $d=3 \mu \mathrm{m}, \Lambda=0.143 \mu \mathrm{m}, a=$ $0.3 \mu \mathrm{m}, n_{2}=3.6, n_{3}=3.4, n_{1}=1$, and took $n_{\mathrm{eff}}=n_{2}$. The midgap wavelength is $\lambda_{0}\left[=\left(2 \pi c / \omega_{0}\right)\right]=1 \mu \mathrm{m}$. The plot corresponding to (109) and (111) is dashed. The solid curves are the result of an "exact" analysis [21].

The filtering properties [22] described above are illustrated in Fig. 8 which is a plot of the intensity transmission $\left|B^{+}(L) / B(0)\right|^{2}$ and reflection $\left|A^{-}(0) / B(0)\right|^{2}$ as obtained from (12). The curves are plotted for $\kappa L=1.84$. The abscissa $\Delta L$ can also be approximated as above by $\Delta L \approx$ 
$\left[\left(\omega n_{2} / c\right)-\eta / 2\right] L$. Fig. 8 thus describes the transmission as a function of frequency (filtering) or, alternatively; as a function of the index $n_{2}$. The latter can be tuned electrooptically, by temperature, photoelastically, or, in anisotropic media, by varying the propagation direction, thus offering some new possibilities for modulation and control.

\section{Mode Coupling by Waveguide ANISOTROPY-MAGNETOOPTIC COUPLING}

If we examine the treatment of mode coupling by the electrooptic of photoelastic effects in Sections V and VII, we find that the coupling between TE and TM modes takes place whenever the dielectric tensor, expressed in terms of the waveguide-coordinate system $x, y, z$, has offdiagonal elements $\epsilon_{x y}$ and $\epsilon_{z y}$. These off-diagonal elements can be induced by an external agency such as a sound wave or applied field. They can, however, be due to deviations of the waveguide material or its orientation from that which was assumed in deriving the behavior of the uncoupled TE and TM modes. In this case we can still describe the propagation in terms of coupling between the unperturbed TE or TM modes. This point of view is fruitful when the initial conditions correspond to either $E_{x}=0$ (TE) or $E_{y}=0(\mathrm{TM})$. We can, alternatively, find the eigenmodes of the "perturbed" system as will be discussed in Section X.

As a demonstration of this point of view we consider the propagation in a dielectric waveguide where one or more of the three layers is magnetic, if the direction of magnetization is parallel to the $z$ direction. The dielectric tensor in the magnetized material is of the form [23]

$$
\tilde{\tilde{\varepsilon}}=\epsilon_{0}\left|\begin{array}{rrr}
\epsilon_{x} & -i \delta & 0 \\
i \delta & \epsilon_{y} & 0 \\
0 & 0 & \epsilon_{z}
\end{array}\right|
$$

Considering the off-diagonal elements of (112) as a perturbation we have

$$
\begin{aligned}
\left(P_{\text {pert }}\right)_{i} & =\epsilon_{i j} E_{i}, & & i \neq j \\
& =0, & & i=j
\end{aligned}
$$

so that a TM input will generate a perturbation polarization

$$
\begin{aligned}
\left(P_{\mathrm{pert}}\right)_{y} & =\epsilon_{y x} \frac{E_{x}^{(\omega)}}{2} \exp \left[i\left(\omega t-\beta_{\mathrm{TM}} z\right)\right]+\text { c.c. } \\
& =i \delta \frac{E_{x}^{(\omega)}}{2} \exp \left[i\left(\omega t-\beta_{\mathrm{TM}} z\right)\right]+\text { c.c. }
\end{aligned}
$$

Using (46) to expand $E_{x}$ in terms of the normal modes $B_{l}{ }^{3} \mathcal{C}_{y}(l)(x),{ }^{5}$ and substituting into the coupled-mode equa-

\footnotetext{
${ }^{5}$ We only keep one term in the expansion (46). This is the term for $\beta_{\mathrm{rM}}^{(l)} \simeq \beta_{\mathrm{TB}}{ }^{(m)}$
}

tion (32) results in

$$
\begin{array}{r}
\frac{d A}{d z}=\frac{\beta_{\mathrm{TM}} B}{4 \epsilon / \epsilon_{0}}\left[\int_{-\infty}^{\infty} \mathcal{H C}_{y}{ }^{(l)}(x) \mathcal{E}_{y}{ }^{(m)}(x) \delta(x, z) d x\right] \\
\cdot \exp \left[i\left(\beta_{\mathrm{TE}}-\beta_{\mathrm{TM}}\right) z\right]
\end{array}
$$

where $A$ is the normal mode amplitude of the $m$ th TE mode, while $B$ is that of the $l$ th TM mode. The offdiagonal element $\delta$ is shown as an explicit function of position. Defining

$$
\kappa=\frac{\beta_{\mathrm{TM}}}{4 \epsilon / \epsilon_{0}} \int_{-\infty}^{\infty} \mathcal{H}_{\nu}{ }^{(l)}(x) \mathcal{E}_{\nu}{ }^{(m)}(x) \delta(x, z) d x
$$

the coupled-mode equations become

$$
\begin{aligned}
\frac{d A}{d z} & =\kappa B e^{i \Delta z} \\
\frac{d B}{d z} & =-\kappa A e^{-i \Delta z} \\
\Delta & \equiv \beta_{\mathrm{TE}}-\beta_{\mathrm{TM}} .
\end{aligned}
$$

The solutions of (117) correspond to the by now familiar case of codirectional exchange as given by (6).

As an example, we calculate $\kappa$ for the case where the guiding layer is paramagnetic, and where the two coupled modes are similar $(l=m)$ and are well above cutoff.

In a paramagnetic material the element $\delta$ is proportional to the applied magnetic field $H$ [22]

$$
\delta=\frac{\lambda_{0} n V}{\pi} H
$$

where $V$ is the Verdet constant of the material and where, in order to limit our attention to the magnetic effect, we take $\epsilon_{x}=\epsilon_{y}=\epsilon_{z} \equiv \epsilon_{0} n^{2}$. Well above cutoff we use (69) to evaluate the integral inside the square brackets of (115).

$\int_{\infty}^{\infty} \mathfrak{K}_{\nu}{ }^{(l)}(x) \mathcal{E}_{\nu}{ }^{(l)}(x) \delta(x, z) d x \approx \delta \int_{0}^{-t} \mathfrak{F}_{\nu}{ }^{(l)} \mathcal{E}_{\nu}{ }^{(l)} d x=2 \delta$.

Using this result in (116)

$$
\kappa=\frac{\pi \delta}{n \lambda_{0}}=V H
$$

In case of phase-matched operation $(\Delta=0)$ with pure TM input, the solution of (117) is

$$
\begin{aligned}
& A=B_{0} \sin \kappa z \\
& B=B_{0} \cos \kappa z .
\end{aligned}
$$

Since $\Delta\left(\equiv \beta_{\mathrm{TE}}-\beta_{\mathrm{TM}}\right)$ is usually different from zero, some means for phase matching is necessary. One solution [24] is to reverse periodically the direction of $H$ with a period $2 \pi / \Delta$. This method, which is analogous to that described 
in Section VI, gives rise to a phase-matched operation with an effective coupling constant which, under square-wave variation of $H$, is smaller by a factor of $2 / \pi$ than that given by (119).

\section{The Eigenmodes of a Perturbed Waveguide}

Up to this point we adopted the point of view of a perturbation that couples the otherwise uncoupled TE and TM modes of a waveguide. This coupling, in the codirectional case, was shown on a number of occasions throughout this paper to lead to equations of the form

$$
\begin{aligned}
& \frac{d A}{d z}=\kappa B e^{-i \Delta z} \\
& \frac{d B}{d z}=-\kappa^{*} A e^{i \Delta z} .
\end{aligned}
$$

We recall that $A$ and $B$ are the normalized mode amplitudes and that the corresponding field variables vary as

$$
\begin{aligned}
& a(x, z, t) \propto A(z) \mathcal{E}_{\nu}{ }^{(m)}(x) e^{i\left(\omega_{a} t-\beta_{a} z\right)} \\
& b(x, z, t) \propto B(z) \mathcal{G}_{\nu}{ }^{(l)}(x) e^{i\left(\omega_{b} t-\beta_{b z}\right)} .
\end{aligned}
$$

An alternative point of view is to find the eigenmodes of the perturbed system, i.e., those linear combinations of $A(z)$ and $B(z)$ which, except for a propagation factor exp $(i \gamma z)$, are independent of $z$.

We define a column vector $\tilde{E}$ as

$$
\tilde{E}(z)=\left|\begin{array}{l}
B(z) e^{-i \beta_{b} z} \\
A(z) e^{-i \beta_{a} z}
\end{array}\right| \equiv\left|\begin{array}{c}
E_{1}(z) \\
E_{2}(z)
\end{array}\right| .
$$

The evolution of $\tilde{E}(z)$ is obtained from (121) and is described by

$$
\frac{d \tilde{E}}{d z}=\tilde{\widetilde{C}} \tilde{E}
$$

with

$$
\approx \widetilde{\widetilde{C}}=\left|\begin{array}{cc}
-i \beta_{b} & -\kappa^{*} \\
\kappa & -i \beta_{a}
\end{array}\right| .
$$

An eigenmode of the waveguide will have a solution of the form

$$
\tilde{E}(z)=\tilde{E}(0) \exp (i \gamma z)
$$

Substituting this form in (124) leads to two homogeneous equations for $E_{1}$ and $E_{2}$

$$
\begin{aligned}
-i\left(\beta_{b}+\gamma\right) E_{1}-\kappa^{*} E_{2} & =0 \\
\kappa E_{1}-i\left(\beta_{a}+\gamma\right) E_{2} & =0 .
\end{aligned}
$$

The solution of the resulting determinantal equation is

$$
\begin{aligned}
\gamma_{1,2} & =-\frac{\left(\beta_{a}+\beta_{b}\right)}{2} \pm \frac{1}{2} \sqrt{\left(\beta_{a}-\beta_{b}\right)^{2}+4 \kappa^{2}} \\
& =-\bar{\beta} \pm \frac{S}{2}
\end{aligned}
$$

$\bar{\beta} \equiv\left(\beta_{a}+\beta_{b}\right) / 2, S \equiv \sqrt{\Delta^{2}+4 \kappa^{2}}, \Delta \equiv \beta_{a}-\beta_{b}$.

The corresponding eigenvectors are found from (126)

$$
\begin{aligned}
& \tilde{E}_{1}=\left|\begin{array}{c}
\frac{2 i \kappa^{*}}{\Delta+S} \\
1
\end{array}\right| e^{-i[\bar{\beta}-(S / 2)\} z} \\
& \tilde{E}_{2}=\left|\begin{array}{c}
2 i \kappa^{*} \\
\Delta-S
\end{array}\right| e^{-i \mid \bar{\beta}+(S / 2)] z} .
\end{aligned}
$$

Note that $\tilde{E}_{1} \cdot \tilde{E}_{1}{ }^{*}$ and $\tilde{E}_{2} \cdot \tilde{E}_{2}{ }^{*}$ are the mode powers and that $\tilde{E}_{1} \cdot \tilde{E}_{2}{ }^{*}=0$. The two components $2 i \kappa^{*} /(\Delta \pm S)$ and 1 of each eigenvector represent the normalized amplitudes of the TE and TM components of each mode, so that the amount of admixture, i.e., the ratio of the powers in the two polarizations, is $4 \kappa^{2} /(\Delta \pm S)^{2}$. In the limit of $\kappa / \Delta \rightarrow 0$, $\tilde{E}_{1}$ and $\tilde{E}_{2}$ become

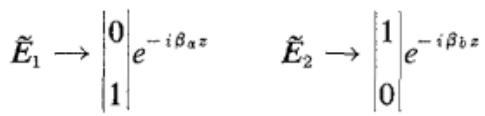

to within a multiplicative constant, and the eigenvectors become the uncoupled TE and TM modes. Another important consequence is that when $\Delta=0, S=2 \kappa$ and the admixture is $50-50$ percent, regardless of $\kappa$. Even a more unconventional consequence is the fact that the two components $E_{1}$ and $E_{2}$ of each eigenvector do not, according to (122), correspond necessarily to fields of the same frequency. It is thus possible, as an example, when the perturbation is time periodic, to have an eigenmode traveling in a waveguide with

$$
\left.E_{x} \propto 2 i \kappa^{*} /(\Delta+S) \exp \left\{i\left[\omega_{b} t-\left(\bar{\beta}-\frac{S}{2}\right)\right]\right]\right\} \mathcal{H C}_{\nu}{ }^{(i)}(x)
$$

while

$$
E_{y} \propto \exp \left\{i\left[\omega_{a} t-\left(\bar{\beta}-\frac{S}{2}\right) z\right]\right\} \varepsilon_{y}{ }^{(m)}(x) .
$$

This is the case in photoelastic coupling by a traveling sound wave discussed in Section VII. What makes these strange bedfellows into a mode is the fact that they travel with the same phase factor, $\exp [-i(\beta-(S / 2)) z]$. This phenomenon may be important in a laser oscillator which contains in its optical path a time-modulated coupling section.

Let us, as an example, apply (129) to the case of magnetooptic coupling as discussed in Section IX. For this 
case we have from (119) $\kappa=V H$, and the eigenmodes (129) in the case $\Delta=0$ become

$$
\begin{aligned}
& \tilde{E}_{1}(z)=\left|\begin{array}{c}
i \\
1
\end{array}\right| e^{-i(\beta-V H) z} \\
& \tilde{E}_{2}(z)=\left|\begin{array}{c}
-i \\
1
\end{array}\right| e^{-i(\beta+V H) z}
\end{aligned}
$$

i.e., the well-known [22] circularly polarized modes. The angle of Faraday rotation in a distance $z$ is

$$
\theta(z)=V H z
$$

\section{Summary}

We have applied the formalism of coupled modes to describe a wide range of experimental situations encountered in guided-wave optics. Explicit expressions for the coupling coefficients, which play a central role in this theory, are given. The formalism treats the case of slab dielectric waveguides, thus assuming no variation in one (y) direction. The extension to guides where the confinement is in both the $x$ and $y$ directions principally involves replacing the integration over all $x$ in the expressions for the coupling coefficients by an integration over both $x$ and $y$. For cases where the modes are very well confined in the $y$ direction, the numerical correction is small.

We have not discussed the applications of the coupledmode formalism to the distributed feedback laser [25], [26] and to directional coupling [27] since the original treatments are already cast in this form.

\section{ACKNOWLEDGMENT}

The author wishes to thank his colleagues, Dr. E. Garmire, Dr. K. Sakuda, and Dr. M. Nakamura, and his students, A. Gover, S. Somekh, and H. Stoll, for their numerous discussions and suggestions.

\section{REFERENCES}

[1] A. Yariv and R. C. C. Leite, "Dielectric waveguide mode of light propagation in p-n junctions," Appl. Phys. Lett., vol. 2, p. 55, 1963.

[2] H. Osterberg and L. W. Smith, "Transmission of optical energy along surfaces," J. Opt. Soc. Amer., vol. 54, p. 1073, 1964.

[3] R. Shubert and J. H. Harris, "Optical surface waves on thin films and their application to integrated data processors," IEEE Trans.
Microwave Theory Tech. (1968 Symposium Issue), vol. MTT-16, pp. 1048-1054, Dec. 1968.

[4] S. E. Miller, "Integrated optics, an introduction,"' Bell Syst. Tech. J. vol. 48 , p. 2059, 1969.

[5] D. F. Nelson and R. K. Rinehart, "Light modulation by the electrooptic effect in reversed-biased GaP functions," Appl. Phys. Lett., vol. 5, p. 148, 1964.

[6] D. Hall, A. Yariv, and E. Garmire, "Optical guiding and electrooptic modulation in GaAs epitaxial layers," Opt. Commun., vol. 1, p. 403, 1970.

[7] D. B. Anderson and J. T. Boyd, "Phase matched second harmonic generation in GaAs thin film waveguides,"Appl. Phys. Lett., vol. 19, p. 266,1971 .

[8] J. R. Pierce, "Coupling of modes of propagation," J. Appl. Phys., vol. 25 , p. 179,1954 .

[9] A. Yariv, "On the coupling coefficients in the coupled-mode theory," Proc. IRE (Corresp.); vol. 46, pp. 1956-1957, Dec. 1958.

[10] A. Gover, private communication.

[11] D. Marcuse, "Mode conversion caused by surface imperfections of a dielectric slab waveguide," vol. 48, p. 3187, 1969.

[12] M. L. Dakss, L. Kuhn, P. F. Heidrich, and B. A. Scott, "Grating coupler for efficient excitation of optical guided waves in thin films," Appl. Phys. Lett., vol. 16, p. 523, 1970.

[13] A. Yariv, Introduction to Optical Electronics. New York: Holt, Rinehart and Winston, 1971, p. 190.

[14] S. Somekh and A. Yariv, "Phase matching by periodic modulation of the nonlinear optical properties," Opt. Commun., vol. 6, p. 301, 1972.

[15] See [13], ch. 9

[16] J. F. Nye, Physical Properties of Crystals. New York: Oxford University Press, 1957.

[17] L. Kuhn, M. L. Dakss, P. F. Heidrich, and B. A. Scott, "Deflection of optical guided waves by a surface acoustic wave," Appl. Phys. Lett., vol. 17 , p. $265,1970$.

[18] R. W. Dixon, "The photoelastic properties of selected materials and their relevance to acoustic light modulators and scanners," J. Appl. Phys., vol. 38, p. 5149, 1967.

[19] A. Yariv, "Quantum theory for parametric interactions of light and hypersound," IEEE J. Quantum Electron., vol. QE-1, pp. 28-36, Apr. 1965.

[20] C. Kittel, Introduction to Solid State Physics. New York: Wiley, 4th ed., ch. 9.

[21] K. Sakuda and A. Yariv, "Analysis of optical propagation in a corrugated dielectric waveguide," Opt. Commun., vol. 8, p. 1, 1973.

[22] F. W. Dabby, A. Kestenbaum, and U. C. Paek, "Periodic dielectric waveguides," Opt. Commun., vol. 6, p. 125, 1972; also F. W. Dabby, M. A. Saifi, and A. Kestenbaum, "High frequency cutoff periodic dielectric waveguides," Appl. Phys. Lett., vol. 22, p. 190, 1973.

[23] W. J. Tabor, "Magnetooptic Materials" in Laser Handbook, F. T. Arecchi and E. O. Schulz-Dubois, Eds. Amsterdam, The Netherlands: North-Holland, 1972.

[24] P. K. Tien, R. J. Martin, S. L. Blank, S. H. Wemple, and L. J. Varnerin, "Optical waveguides in single crystal garnet films," Appl. Phys. Lett., vol. 21, p. 207, 1972.

[25] H. Kogelnik and C. V. Shank, "Coupled wave theory of distributed feedback lasers," J. Appl. Phys., vol. 43, p. 2328, 1972.

[26] P. Zory, "Laser oscillation in corrugated leaky optical waveguides," to be published.

[27] S. Somekh, E. Garmire, A. Yariv, H. L. Garvin, and R. G. Hunsperger, "Channel optical waveguide directional couplers," Appl. Phys. Lett., vol. 22, p. 46, 1972. 